\title{
Almost Hermitian Identities
}

\author{
Joana Cirici ${ }^{1}$ and Scott $\mathrm{O}$. Wilson ${ }^{2, *}$ \\ 1 Department of Mathematics and Computer Science, Universitat de Barcelona, Gran Via 585, \\ 08007 Barcelona, Spain; jcirici@ub.edu \\ 2 Department of Mathematics, Queens College, City University of New York, 65-30 Kissena Blvd., \\ Flushing, NY 11367, USA \\ * Correspondence: scott.wilson@qc.cuny.edu
}

Received: 30 July 2020; Accepted: 11 August 2020; Published: 13 August 2020

check for updates

\begin{abstract}
We study the local commutation relation between the Lefschetz operator and the exterior differential on an almost complex manifold with a compatible metric. The identity that we obtain generalizes the backbone of the local Kähler identities to the setting of almost Hermitian manifolds, allowing for new global results for such manifolds.
\end{abstract}

Keywords: almost Hermitian manifolds; Kähler identities; Lefschetz operator

\section{Introduction}

On a Kähler manifold $(M, J, \omega)$, the most fundamental local identity is perhaps the commutation relation between the exterior differential $d$ and the adjoint $\Lambda$ to the Lefschetz operator,

$$
[\Lambda, d]=\star \mathbb{I}^{-1} d \mathbb{I} \star,
$$

where $\star$ denotes the Hodge star operator and $\mathbb{I}$ denotes the extension of $J$ to all forms.

This identity, due to A. Weil [1], strongly depends on the Kähler condition, $d \omega=0$, and in fact is true when removing the integrability condition $N_{J} \equiv 0$. So, it is valid for almost Kähler and also symplectic manifolds as well [2-4]. On the other hand, there is also a generalization of the Kähler identities in the Hermitian setting (see [5,6]), which strongly uses integrability.

When the manifold is only almost Hermitian, then the above local identity does not hold in general, as noticed implicitly in [7]. The purpose of this short note is to show precisely how the above Kähler identity (1) becomes modified when the form $\omega$ is not closed.

The main result is given in Theorem 1 below, which has several applications including the uniqueness of the Dirichlet problem

$$
\partial \bar{\partial} u=g \quad \text { with }\left.\quad u\right|_{\partial \Omega}=\phi,
$$

on any compact domain $\Omega$ in an almost complex manifold. This in turn implies that the Dolbeault cohomology introduced in [8], for all almost complex manifolds, satisfies $H_{\text {Dol }}^{0,0}(M) \cong \mathbb{C}$ for a compact connected almost complex manifold.

Another application of the almost Hermitian identities of Theorem 1 appears in forthcoming work by Feehan and Leness [9]. There the fundamental relation of Proposition 1 is used to show that the moduli spaces of unitary anti-self-dual connections over any almost Hermitian 4-manifold is almost Hermitian, whenever the Nijenhuis tensor has sufficiently small $C^{0}$-norm. This generalizes a well known result for Kähler manifolds that was exploited in Donaldson's work in the 1980s, and is expected to have consequences for the topology of almost complex 4-manifolds which are of so-called Seiberg-Witten simple type. 
When $M$ is compact, local identities lead to consequences in cohomology, often governed by geometric-topological inequalities. Indeed, the exterior differential inherits a bidegree decomposition into four components $d=\bar{\mu}+\partial+\partial+\mu$ and the Hermitian metric allows one to consider the Laplacian operators associated to each of these components. In the compact case, the numbers

$$
\ell^{p, q}:=\left.\operatorname{dim} \operatorname{Ker}\left(\Delta_{\bar{\jmath}}+\Delta_{\mu}\right)\right|_{(p, q)}
$$

given by the kernel of $\Delta_{\bar{\jmath}}+\Delta_{\mu}$ in bidegree $(p, q)$ are finite by elliptic operator theory. When $J$ is integrable (and so $M$ is a complex manifold) the operator $\Delta_{\mu}$ vanishes and these are just the Hodge numbers $\ell^{p, q}=h^{p, q}$. In this case, the Hodge-to-de Rham spectral sequence gives inequalities

$$
\sum_{p+q=k} \ell^{p, q} \geq b^{k}
$$

where $b^{k}$ denotes the $k$-th Betti number. On the other hand, as shown in [4], one main consequence of the local identity (1) in the almost Kähler case $d \omega=0$ is the converse inequality

$$
\sum_{p+q=k} \ell^{p, q} \leq b^{k}
$$

Of course, in the integrable Kähler case both inequalities are true and so one recovers the well-known consequence of the Hodge decomposition

$$
\sum_{p+q=k} \ell^{p, q}=b^{k}
$$

The local identities of [5,6] for complex non-Kähler manifolds include other algebra terms which lead to further Laplacian operators, leading also to various inequalities relating the geometry with the topology of the manifold.

With this note, we aim to further understand the origin of these inequalities by means of the correct version of (1) for almost Hermitian manifolds for which, a priori, the only geometric-topological inequality in the compact case is given by

$$
\left.\sum_{p+q=k} \operatorname{dim} \operatorname{Ker}\left(\Delta_{\bar{\mu}}+\Delta_{\bar{\partial}}+\Delta_{\partial}+\Delta_{\mu}\right)\right|_{(p, q)} \leq b^{k}
$$

\section{Preliminaries}

Let $(\mathcal{A}, d)$ denote the complex valued differential forms of an almost complex manifold $(M, J)$. For any Hermitian metric, define the associated Hodge-star operator

$$
\star: \mathcal{A}_{x}^{p, q} \rightarrow \mathcal{A}_{x}^{n-q, n-p} \text { by } \omega \wedge \star \bar{\eta}=\langle\omega, \eta\rangle \mathrm{vol},
$$

where $\omega$ is the fundamental $(1,1)$-form, and vol $=\frac{1}{n !} \omega^{n} \in \mathcal{A}^{n, n}$ is the volume form determined by the Hermitian metric. Note $\star^{2}=(-1)^{k}$ on $\mathcal{A}^{k}$.

Define $d^{*}=-\star d \star$, so that $d^{*} \star=(-1)^{k+1} \star d$ on $\mathcal{A}^{k}$. Similarly, consider the bidegree decomposition of the exterior differential

$$
d=\bar{\mu}+\bar{\partial}+\partial+\mu,
$$

where the bidegree of each component is given by

$$
|\bar{\mu}|=(-1,2),|\bar{\partial}|=(0,1),|\partial|=(1,0) \text { and }|\mu|=(2,-1) .
$$


We then let $\bar{\delta}^{*}=-\star \delta \star$ for $\delta=\bar{\mu}, \bar{\partial}, \partial, \mu$ and we have the bidegree decomposition

$$
d^{*}=\bar{\mu}^{*}+\bar{\partial}^{*}+\partial^{*}+\mu^{*} .
$$

where

$$
\left|\bar{\mu}^{*}\right|=(1,-2),\left|\bar{\partial}^{*}\right|=(0,-1),\left|\partial^{*}\right|=(-1,0) \text { and }\left|\mu^{*}\right|=(-2,1) .
$$

Let $L: \mathcal{A}^{p, q} \rightarrow \mathcal{A}^{p+1, q+1}$ be the real $(1,1)$-operator given by $L(\eta)=\omega \wedge \eta$. Let $\Lambda=L^{*}=\star^{-1} L \star$. Then $\star \Lambda=L \star$ and $\star L=\Lambda \star$. Let $P^{k}=\operatorname{Ker} \Lambda \cap \mathcal{A}^{k}$ denote the primitive forms of total degree $k$.

It is well known that $\{L, \Lambda,[L, \Lambda]\}$ defines a representation of $\operatorname{sl}(2, \mathbb{C})$ and induces the Lefschetz decomposition on forms:

Lemma 1. We have

$$
\mathcal{A}^{k}=\bigoplus_{r=0}^{k / 2} L^{r} P^{k-2 r},
$$

and this direct sum decomposition respects the $(p, q)$ bigrading.

Let $[A, B]=A B-(-1)^{|A||B|} B A$ be the graded commutator, where $|A|$ denotes the total degree of $A$. This defines a graded Poisson algebra

$$
[A, B C]=[A, B] C+(-1)^{|A||B|} B[A, C]
$$

The following is well known (e.g., [10] Corollary 1.2.28):

Lemma 2. For all $j \geq 0$ and $\alpha \in \mathcal{A}^{k}$

$$
\left[L^{j}, \Lambda\right] \alpha=j(k-n+j-1) L^{j-1} \alpha .
$$

By induction, and the fact that $[d, L]$ and $L$ commute, we have:

Lemma 3. For all $n \geq 1$

$$
\left[d, L^{n}\right]=n[d, L] L^{n-1},
$$

and

$$
\star[d, L] \alpha=(-1)^{k+1}\left[d^{*}, \Lambda\right] \star \alpha \quad \text { for } \alpha \in \mathcal{A}^{k} .
$$

Let $\mathbb{I}$ be the extension of $J$ to all forms as an algebra map with respect to wedge product, so that $\mathbb{I}_{p, q}$ acts on $\mathcal{A}^{p, q}$ by multiplication by $i^{p-q}$. Then $\mathbb{I}_{p, q}^{2}=(-1)^{p+q}$ so that $\mathbb{I}_{p, q}^{-1}=(-1)^{p+q} \mathbb{I}_{p, q}$. Note that $\mathbb{I}$ and $\star$ commute, and $\mathbb{I}$ and $L^{n}$ commute for all $n \geq 0$. The following is a direct calculation.

Lemma 4. If an operator $T_{r, s}: \mathcal{A}^{p, q} \rightarrow \mathcal{A}^{p+r, q+s}$ has bidegree $(r, s)$, then

$$
\mathbb{I}_{r+p, s+q}^{-1} \circ T_{r, s} \circ \mathbb{I}_{p, q}=(-i)^{r-s} T_{r, s} .
$$

The above result readily implies that

$$
\mathbb{I}^{-1} \circ d \circ \mathbb{I}=-i(\bar{\mu}-\bar{\partial}+\partial-\mu) .
$$

Finally, the following is well known (e.g., [10] Proposition 1.2.31):

Lemma 5. If $M$ is an almost Hermitian manifold of dimension $2 n$, then for all $j \geq 0$ and all $\alpha \in P^{k}$,

$$
\star L^{j} \alpha=(-1)^{\frac{k(k+1)}{2}} \frac{j !}{(n-k-j) !} L^{n-k-j} \mathbb{I} \alpha .
$$




\section{Almost Hermitian Identities}

By the previous section, any differential form $\eta$ can be written as $\eta=L^{j} \alpha$ for unique $j, k \geq 0$ and $\alpha \in P^{k}$. We now state the main result:

Theorem 1. For any almost Hermitian manifold of dimension $2 n$, let $\alpha \in P^{k}$, with $d \alpha$ written as

$$
d \alpha=\alpha_{0}+L \alpha_{1}+L^{2} \alpha_{2}+\cdots
$$

for unique $\alpha_{r} \in P^{k+1-2 r}$. Then, for all $j \geq 0$,

$$
\begin{aligned}
{[\Lambda, d] L^{j} \alpha-\star \mathbb{I}^{-1} d \mathbb{I} \star L^{j} \alpha } & =\frac{1}{j+1} \mathbb{I}^{-1}\left[d^{*}, \Lambda\right] \mathbb{I} L^{j+1} \alpha \\
& +j \Lambda[d, L] L^{j-1} \alpha+j(j-1)(k-n+j-1)[d, L] L^{j-2} \alpha \\
& +\sum_{r=2}^{\infty} f_{n, j, k}(r) L^{j+r-1} \alpha_{r}
\end{aligned}
$$

where

$$
f_{n, k, j}(r)=(r(n-k+r)-j)+(-1)^{r} \frac{j !(n-k-j+r) !}{(j+r-1) !(n-k-j) !} .
$$

Remark 1. In the almost Kähler case we have $\left[d^{*}, \Lambda\right]=[d, L]=0$, and $d \alpha=\alpha_{0}+L \alpha_{1}$, so we recover the identity

$$
[\Lambda, d]=\star \mathbb{I}^{-1} d \mathbb{I} \star
$$

as expected.

Proof. The proof consists of several calculations using the lemmas in the previous section. Using $[\mathbb{I}, L]=0$, and $\mathbb{I}^{2}=(-1)^{k}$ on $\mathcal{A}^{k}$, we have

$$
\begin{aligned}
\star \mathbb{I}^{-1} d \mathbb{I} \star \eta & =\star \mathbb{I}^{-1} d \mathbb{I}\left((-1)^{\frac{k(k+1)}{2}} \frac{j !}{(n-k-j) !} L^{n-k-j} \mathbb{I} \alpha\right) \\
& =(-1)^{\frac{k(k+1)}{2}}+k \frac{j !}{(n-k-j) !} \star \mathbb{I}^{-1} d L^{n-k-j} \alpha .
\end{aligned}
$$

By Lemma 3 this is equal to

$$
(-1)^{\frac{k(k+1)}{2}+k} \frac{j !}{(n-k-j) !} \star \mathbb{I}^{-1} L^{n-k-j} d \alpha+(-1)^{\frac{k(k+1)}{2}+k} \frac{j !}{(n-k-j-1) !} \star \mathbb{I}^{-1}[d, L] L^{n-k-j-1} \alpha .
$$

We first simplify each of these last two summands. By Equation (2), the fact that $\star$ commutes with $\mathbb{I}$, and Lemma 5 applied to $\alpha_{r} \in P^{k+1-2 r}$, the first summand of Equation (3) is equal to:

$$
\begin{aligned}
&(-1)^{\frac{k(k+1)}{2}+k} \frac{j !}{(n-k-j) !} \star \mathbb{I}^{-1}\left(\sum_{r=0}^{\infty} L^{n-k-j+r} \alpha_{r}\right) \\
&=(-1)^{\frac{k(k+1)}{2}+k} \frac{j !}{(n-k-j) !} \mathbb{I}^{-1}\left(\sum_{r=0}^{\infty}(-1)^{\frac{(k+1-2 r)(k-2 r+2)}{2}} \frac{(n-k-j+r) !}{(j+r-1) !} L^{j+r-1} \mathbb{I} \alpha_{r}\right) \\
&=\sum_{r=0}^{\infty}(-1)^{r+1} \frac{j !(n-k-j+r) !}{(j+r-1) !(n-k-j) !} L^{j+r-1} \alpha_{r} .
\end{aligned}
$$

For the second summand, we use the fact that for all $m \geq 0$ and $\beta \in \mathcal{A}^{k}$,

$$
\star L^{m}[d, L] \beta=\star[d, L] L^{m} \beta=(-1)^{k+1}\left[d^{*}, \Lambda\right] \star L^{m} \beta .
$$


So, the second summand in Equation (3) is equal to

$$
\begin{gathered}
(-1)^{\frac{k(k+1)}{2}+k} \frac{j !}{(n-k-j-1) !} \star \mathbb{I}^{-1}[d, L] L^{n-k-j-1} \alpha \\
=(-1)^{\frac{k(k+1)}{2}+1} \frac{j !}{(n-k-j-1) !} \mathbb{I}^{-1}\left[d^{*}, \Lambda\right] \star L^{n-k-j-1} \alpha \\
=(-1)^{\frac{k(k+1)}{2}+1} \frac{j !}{(n-k-j-1) !} \mathbb{I}^{-1}\left[d^{*}, \Lambda\right](-1)^{\frac{k(k+1)}{2} \frac{(n-k-j-1) !}{(j+1) !} L^{j+1} \mathbb{I} \alpha} \\
=\frac{-1}{j+1} \mathbb{I}^{-1}\left[d^{*}, \Lambda\right] \mathbb{I} L^{j+1} \alpha,
\end{gathered}
$$

where in the second to last step we used Lemma 5.

In summary, we have

$$
\star \mathbb{I}^{-1} d \mathbb{I} \star \eta=\sum_{r=0}^{\infty}(-1)^{r+1} \frac{j !(n-k-j+r) !}{(j+r-1) !(n-k-j) !} L^{j+r-1} \alpha_{r}-\frac{1}{j+1} \mathbb{I}^{-1}\left[d^{*}, \Lambda\right] \mathbb{I} L^{j+1} \alpha .
$$

We now compute $[\Lambda, d] \eta$, by first computing $\Lambda d L^{j} \alpha$, using that all $\alpha_{r}$ are primitive. By Equation (2), Lemma 2, and Lemma 3, we have:

$$
\begin{aligned}
\Lambda d L^{j} \alpha & =\Lambda L^{j} d \alpha+\Lambda\left[d, L^{j}\right] \alpha \\
& =\Lambda L^{j}\left(\sum_{r=0}^{\infty} L^{r} \alpha_{r}\right)+j \Lambda[d, L] L^{j-1} \alpha \\
& =\sum_{r=0}^{\infty} \Lambda L^{j+r} \alpha_{r}+j \Lambda[d, L] L^{j-1} \alpha \\
& =-\sum_{r=0}^{\infty}(j+r)(k+1-2 r-n+j+r-1) L^{j+r-1} \alpha_{r}+j \Lambda[d, L] L^{j-1} \alpha .
\end{aligned}
$$

Next using, $\alpha$ is primitive, and Lemma 2 again, we have

$$
\begin{aligned}
d \Lambda L^{j} \alpha & =-j(k-n+j-1) d L^{j-1} \alpha \\
& =-j(k-n+j-1) L^{j-1} d \alpha-j(k-n+j-1)(j-1)[d, L] L^{j-2} \alpha \\
& =-j(k-n+j-1)\left(\sum_{r=0}^{\infty} L^{j+r-1} \alpha_{r}\right)-j(k-n+j-1)(j-1)[d, L] L^{j-2} \alpha .
\end{aligned}
$$

So,

$$
[\Lambda, d] \eta=\sum_{r=0}^{\infty}(r(n-k+r)-j) L^{j+r-1} \alpha_{r}+j \Lambda[d, L] L^{j-1} \alpha+j(j-1)(k-n+j-1)[d, L] L^{j-2} \alpha .
$$

Using this last equation and combining with Equation (4) we obtain the desired result:

$$
\begin{aligned}
{[\Lambda, d] \eta-\star \mathbb{I}^{-1} d \mathbb{I} \star \eta } & =\frac{1}{j+1} \mathbb{I}^{-1}\left[d^{*}, \Lambda\right] \mathbb{I} L^{j+1} \alpha \\
& +j \Lambda[d, L] L^{j-1} \alpha+j(j-1)(k-n+j-1)[d, L] L^{j-2} \alpha \\
& +\sum_{r=0}^{\infty} f_{n, k, j}(r) L^{j+r-1} \alpha_{r}
\end{aligned}
$$

where

$$
f_{n, k, j}(r)=(r(n-k+r)-j)+(-1)^{r} \frac{j !(n-k-j+r) !}{(j+r-1) !(n-k-j) !} .
$$


It is a curious fact that $f(0)=f(1)=0$, whereas for $r \geq 2, f(r)$ is in general non-zero.

\section{Applications}

On an almost Kähler manifold, using the bidegree decompositions of $d$ and $d^{*}$, one may derive from (1) the relation

$$
[\Lambda, \partial]=i \bar{\partial}^{*},
$$

involving $\Lambda, \partial$ and the adjoint of $\bar{\partial}$. For a non-Kähler Hermitian manifold there is an additional term

$$
[\Lambda, \partial]=i\left(\bar{\partial}^{*}+\bar{\tau}^{*}\right)
$$

where $\bar{\tau}=[\Lambda,[\bar{\partial}, L]]$ is the zero-order torsion operator (see $[5,6])$. In the case of $(0, q)$-forms this gives

$$
\Lambda \partial \alpha=i \bar{\partial}^{*} \alpha+i\left[\Lambda, \bar{\partial}^{*}\right] L \alpha
$$

Next we use Theorem 1 to derive this local identity also in the non-integrable case.

Proposition 1. For all $\alpha \in \mathcal{A}^{0, q}$ in an almost Hermitian manifold we have

$$
\Lambda \partial \alpha=i \bar{\partial}^{*} \alpha+i\left[\Lambda, \bar{\partial}^{*}\right] L \alpha
$$

Proof. By bidegree reasons $\alpha$ is a primitive form and we have $d \alpha=\alpha_{0}+L \alpha_{1}+L^{2} \alpha_{2}$ where $\alpha_{i}$ are primitive. By expanding each term in the equality of Theorem 1 with respect to the bidegree decomposition $d=\bar{\mu}+\bar{\partial}+\partial+\mu$, in the case $j=0$, we obtain:

$$
\begin{aligned}
& {[\Lambda, d] \alpha=\Lambda d \alpha=\Lambda(\partial+\mu) \alpha,} \\
& \star \mathbb{I}^{-1} d \mathbb{I} \star \alpha=i\left(\bar{\partial}^{*}-\bar{\mu}^{*}\right) \alpha,
\end{aligned}
$$

and

$$
\mathbb{I}^{-1}\left[d^{*}, \Lambda\right] \mathbb{I} L \alpha=i\left[\Lambda, \bar{\partial}^{*}-\bar{\mu}^{*}\right] L \alpha .
$$

In particular, all terms decompose into sums of pure bidegrees $(0, q-1)$ and $(1, q-2)$. Note as well that the remaining term

$$
f_{n, q, 0}(2) L \alpha_{2}
$$

given in Theorem 1 has pure bidegree $(1, q-2)$, since $\alpha_{2}$ must have bidegree $(0, q-3)$. By putting together all terms of bidegree $(0, q-1)$ we obtain the desired identity.

Remark 2. The proof of Proposition 1 gives a second identity relating the operators $\Lambda, \mu$ and $\bar{\mu}$ and their adjoints, which also contains the term $f_{n, q, 0}(2) L \alpha_{2}$. For forms in $\mathcal{A}^{0,2}$, this extra term vanishes by bidegree reasons, since $\alpha_{2}=0$. Then the second identity reads

$$
\Lambda \mu \alpha=-i \bar{\mu}^{*} \alpha-i\left[\Lambda, \bar{\mu}^{*}\right] L \alpha .
$$

This corrects the identity

$$
[\Lambda, \mu]=-i \bar{\mu}^{*}
$$

known in the almost Kähler case for arbitrary forms (see [4]).

The previous proposition can be used to give a uniqueness result for the Dirichlet problem on compact domains with a boundary. 
Corollary 1 . Let $\Omega$ be a compact domain in an almost complex manifold $(M, J)$, with smooth boundary, and let $g: \Omega \rightarrow \mathbb{C}$, and $\phi: \partial \Omega \rightarrow \mathbb{C}$ be smooth. Then the Dirichlet problem,

$$
\partial \bar{\partial} u=g \quad \text { with }\left.\quad u\right|_{\partial \Omega}=\phi,
$$

has at most one solution $u: \Omega \rightarrow \mathbb{C}$.

In particular, if $(M, J)$ is a compact connected almost complex manifold, and $f: M \rightarrow \mathbb{C}$ is a smooth map of almost complex manifolds, then $f$ is constant.

Proof. It suffices to show the only solution to the homogenous equation with $g=0$ is a constant function.

In any coordinate chart $\psi: V \rightarrow \mathbb{R}^{2 n}$ containing any maximum point, we pullback $J$ to $\psi(V)$ and consider the J-preserving map $u \circ \psi^{-1}: \psi(V) \rightarrow \mathbb{C}$. The components of $d$ are natural with respect to this $J$-preserving map and we use a compatible metric on $\psi(V)$ to define $\Lambda$ and $\bar{\partial}^{*}$. Then by Proposition 1 with $q=1$ we obtain

$$
-i \Lambda \partial \bar{\partial} u=\bar{\partial}^{*} \bar{\partial} u+\left[\Lambda, \bar{\partial}^{*}\right] L \bar{\partial} u
$$

on $\psi(V)$. Note $\bar{\partial}^{*} \bar{\partial}$ is quadratic, self-adjoint, and positive, and $\left[\Lambda, \bar{\partial}^{*}\right] L \bar{\partial}$ is first order since $\left[\Lambda, \bar{\partial}^{*}\right]=$ $[d, L]^{*}$ is zeroth order, because $[d, L] \eta=d \omega \wedge \eta$. Then the right hand side is zero, so the maximum principle due to E. Hopf applies [11], showing $u$ is constant in a neighborhood of the maximum point and therefore, by connectedness, $u$ is constant.

The final claim follows taking $\Omega=M$, with empty boundary, $g=0$, and noting the condition that $f$ is a map of almost complex manifolds implies $\bar{\partial} f=0$.

Remark 3. In [8], we introduce a Dolbeault cohomology theory that is valid for all almost complex manifolds. The above corollary is key in showing that, for a compact connected almost complex manifold, this cohomology is well-behaved in lowest bidegree, in the sense that $H_{\text {Dol }}^{0,0}(M) \cong \mathbb{C}$.

Finally, we refer the reader to the work of Feehan and Leness [9], where the relation of Proposition 1, for $q=1$, is used to show that the moduli spaces of unitary anti-self-dual connections over any almost Hermitian 4-manifold is almost Hermitian, whenever the Nijenhuis tensor has sufficiently small $C^{0}$-norm.

Author Contributions: Conceptualization, J.C. and S.O.W.; Writing-original draft, J.C. and S.O.W.; Writing-review \& editing, J.C. and S.O.W. Both authors have read and agreed to the published version of the manuscript.

Funding: J.C. would like to acknowledge partial support from the AEI/FEDER, UE (MTM2016-76453-C2-2-P) and the Serra Húnter Program. S.O.W. acknowledges the support provided by a PSC-CUNY Award, jointly funded by The Professional Staff Congress and The City University of New York.

Acknowledgments: The authors would like to thank Paul Feehan for encouraging us to develop some previous notes into the present paper.

Conflicts of Interest: The authors declare no conflict of interest.

\section{References}

1. Weil, A. Introduction à l'étude des Variétés Kählériennes; l'Institut de Mathématique de l'Université de Nancago: Hermann, Paris, 1958; Volume VI, p. 175.

2. De Bartolomeis, P.; Tomassini, A. On formality of some symplectic manifolds. Intern. Math. Res. Not. 2001, 1287-1314. [CrossRef]

3. Tardini, N.; Tomassini, A. Differential operators on almost-Hermitian manifolds and harmonic forms. Complex Manifolds 2020, 7, 106-128. [CrossRef] 
4. Cirici, J.; Wilson, S.O. Topological and geometric aspects of almost Kähler manifolds via harmonic theory. Sel. Math. 2020, 26, 35. [CrossRef]

5. Demailly, J.P. Sur l'identité de Bochner-Kodaira-Nakano en géométrie hermitienne. In Séminaire d'analyse P. Lelong-P. Dolbeault-H. Skoda, Années 1983/1984; Springer: Berlin, Germany, 1986; Volume 1198, pp. 88-97.

6. Wilson, S.O. Harmonic symmetries for Hermitian manifolds. Proc. Am. Math. Soc. 2020, 148, 3039-3045. [CrossRef]

7. Ohsawa, T. Isomorphism theorems for cohomology groups of weakly 1-complete manifolds. Publ. Res. Inst. Math. Sci. 1982, 18, 191-232. [CrossRef]

8. Cirici, J.; Wilson, S. Dolbeault cohomology for almost complex manifolds. arXiv 2018, arXiv:1809.01416.

9. Feehan, P.M.N.; Leness, T.G. Virtual Morse theory, almost Hermitian manifolds, SO(3) monopoles, and applications to four-manifold topology. In preparation.

10. Huybrechts, D. Complex Geometry; An Introduction; Springer: Berlin, Germany, 2005; pp. xii, 309.

11. Hopf, E. Selected Works of Eberhard Hopf with Commentaries; Cathleen S., Serrin, M.J.B., Sinai, Y.G., Eds.; American Mathematical Society: Providence, RI, USA, 2002; pp. xxiv, 386.

(C) 2020 by the authors. Licensee MDPI, Basel, Switzerland. This article is an open access article distributed under the terms and conditions of the Creative Commons Attribution (CC BY) license (http://creativecommons.org/licenses/by/4.0/). 